\title{
Post-operative of bleeding, haemolysis and coagulation in mechanical circulatory support patients
}

\author{
Martin W. Besser \\ Department of Haematology, Addenbrooke's Hospital, Cambridge, UK \\ Correspondence to: Martin W. Besser, FRCP, FRCPath. Consultant Haematologist, Royal Papworth Hospital, Box 217, Addenbrooke's Hospital \\ Cambridge CB2 0QQ, UK. Email: Martin.Besser@nhs.net.
}

\begin{abstract}
There are unique complications arising from mechanical support devices but some of the longterm systemic haematological complications are indistinguishable from management problems affecting the care of other patients receiving intermediate to long term care in the cardiac ICU. The field of mechanical cardiac assist device (MCAD) is evolving. Despite major changes in design of these devices the most feared haematological complications have remained unchanged, namely haemolysis, pump thrombosis or thromboembolism. This review article gives an overview over the pathophysiology of MCAD related haematological complications, their management and where possible an outlook on future strategies to prevent such complications. The impact of MCAD on blood is discussed, starting with rheology, common pump mechanisms, current and future pump surface coating materials, anatomical considerations of the connection of the circuit and design of the circuit itself. Moreover, the duration of the cardiovascular support, impact of bleeding complications and other patient factors. This article also covers the impact of long term mechanical cardiac support on the properties of platelets, the anticoagulation strategies and a basic guide to the differential diagnosis of haemolysis is reviewed. The section on anaemia considers anaemia in the wider perioperative setting for patients in critical care having undergone cardiac surgery and also discusses transfusion alternatives.
\end{abstract}

Keywords: Thrombosis; bleeding; anticoagulation; transfusion; extracorporeal membrane oxygenation (ECMO); haemolysis; platelet dysfunction; mechanical cardiac assist device (MCAD)

Submitted Jan 08, 2020. Accepted for publication Apr 29, 2020.

doi: 10.21037/atm-20-405

View this article at: http://dx.doi.org/10.21037/atm-20-405

\section{Introduction}

The field of mechanical cardiac assist device (MCAD) is evolving. Despite major changes in design of these devices the most feared haematological complications have remained unchanged, namely haemolysis, pump thrombosis or thromboembolism. Ventricular assist devices have been used successfully since 1966 and there is now registry data for more than 15,000 implantations $(1,2)$. In the UK in 2019 the average wait for a heart transplant was 3 years (3). The use of MCAD as bridge or destination therapy has improved survival and quality of life.

The current guidelines regarding the implantation and maintenance anticoagulation are based on very small studies, registries and expert opinion.

The implantation of cardiac assist devices typically is performed in patients with coagulopathy and commonly/ thrombocytopenia due to critical illness or right heart failure, but also in patients with advanced heart failure that are no longer critically ill. A number of patients are anaemic and show a degree of iron restriction. Whether these devices are used as a bridge or potential destination therapy, potential HLA sensitisation due to transfusion is a concern and transfusion avoidance is important.

Safe platelet and red cell transfusion thresholds vary between individuals and between adults and children. This review is an overview about the current practice, how to diagnose and approach mechanical assist device induced 


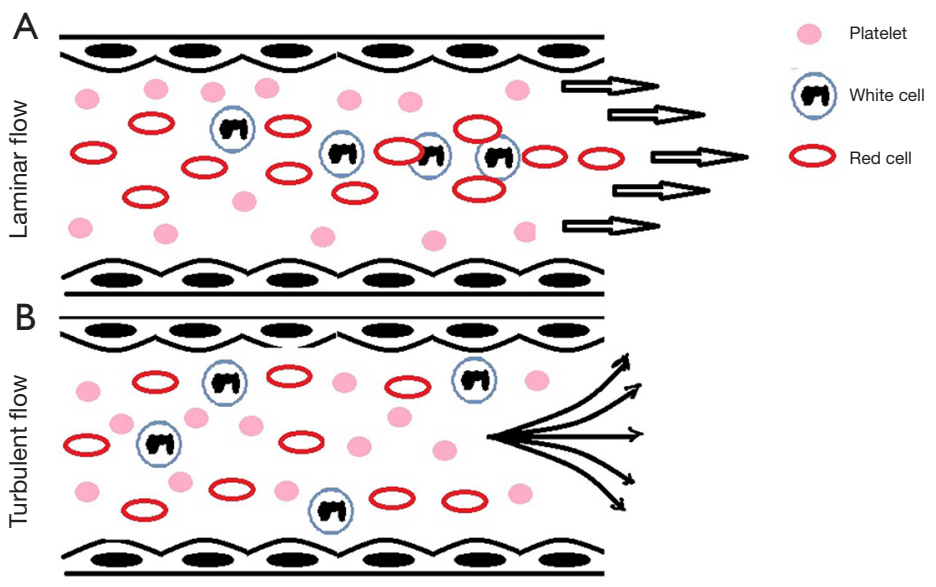

Figure 1 These two panels depict laminar and turbulent flow. Note redistribution of cellular elements across the vascular cross section dependent on flow.

haemolysis, the optimum perioperative transfusion strategy and alternatives to transfusion, current and future strategies to minimise contact activation are also discussed.

\section{Haematological aspects of cardiac assist devices}

To understand the possible range of complications in patients with mechanical assist devices it is important to review the following shared principles and complications which apply to all devices to a varying degree.

\section{Rheology}

Blood's viscosity decreases with increasing shear force (non-Newtonian fluid or 'shear-thinning' fluid). Its compressibility which is caused predominantly by deformability of the red cells. At molecular level this also causes changes in the shape of platelets but also at a tertiary structure level of proteins such as von Willebrand factor (vWF). Red cell breakdown occurs at forces $15 \times$ greater than the force to unfold a $\mathrm{vWF}$ protein and $3 \times$ greater than the force required to activate a platelet. Shear force activated platelets participate in coating the surface of the tubing (4). Under physiological conditions the laminar flow of blood leads to marginalisation of platelets. Turbulent flow interrupts this physiological pattern. When platelets no longer marginalise so thrombocytopenia becomes more symptomatic, as more platelets are required per ul to achieve the same density of platelets in the outer layer close to the endothelium. Blood is more likely to exhibit a turbulent flow as the haematocrit decreases (5).

Figure 1 shows an overview of cell distribution under laminar and turbulent flow conditions.

\section{Pump mechanism}

Blood has evolved to undergo pulsatile flow with gradual deceleration. The introduction of MCAD pumps which maybe pulsatile [e.g., pneumatic (HeartMate ${ }^{\circledR}$, Abbot Chicago, IL, USA)] or continuous [centrifugal (HVAD ${ }^{\circledR}$, HeartWare Int., Framingham, MA, USA) or axial [e.g., via an Archimedian screw (HeartMate II ${ }^{\circledR}$, Abbot, Chicago, IL, USA)]. The majority of the devices currently use continuous flow with either axial or centrifugal pumps. Figure 1 gives an overview of flow patterns with continuous and pulsatile mechanical assist devices. Figure 2 gives an overview of mechanical assist devices (6). Axial flow is defined as a flow pattern that is associated with minimal change of the radial location of fluid particles ('pushing', the axis of flow is in line with the impella axis). In a centrifugal pump blood is moved tangentially and radially leading to a higher degree of turbulence ('throwing', the axis of flow is at 90 degrees to the impella axis) $(7,8)$.

Partial circulatory support can enhance this turbulence effect even further (9).

This classification is made more complex by the ability of some pumps to vary the pressure pattern intermittently (e.g., HeartMate III $^{\circledR}$ ) and variability in the degree of valve opening and closing patterns participating in the 


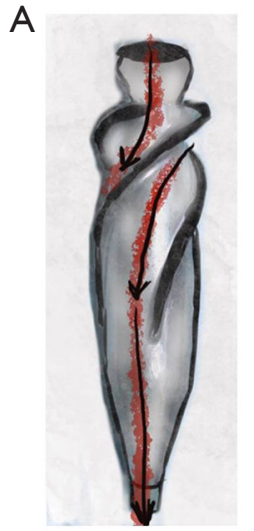

Continuous axial flow

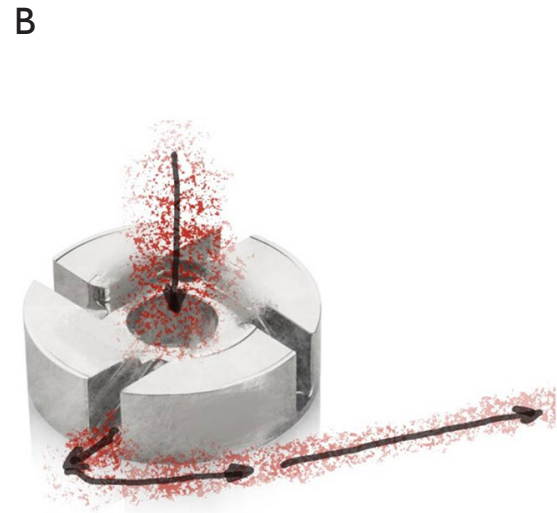

Continuous centrifugal flow

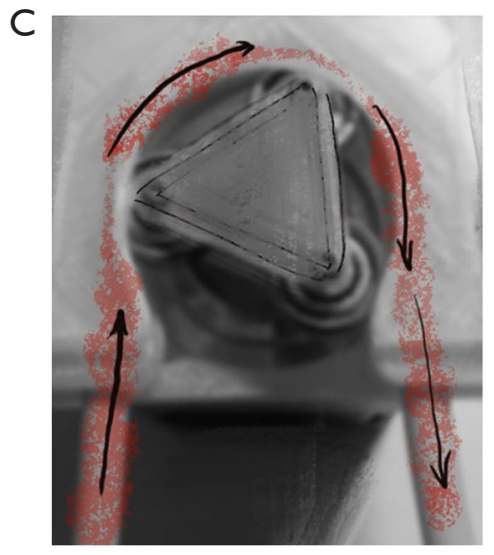

Pulsatile flow

Figure 2 Three current LVAD pump principles. (A) Depicts an axial flow impella such as the one used in the HeartMate II device; (B) depicts a centrifugal pump such as those used in the HVAD ${ }^{\circledR}$ device; $(\mathrm{C})$ depicts the principle of a roller pump such as those used in paracardial devices such as during CPB. Action of the roller pump leads to intermittent marked compression of the blood tube.

modulation of the pressure waves (10).

Areas of sudden deceleration or rapidly changing vascular geometry are sites of depletion particularly for high molecular-weight vWF which can lead to an unexpected discrepancy between circulating high vWF Antigen and vWF Activity [ristocetin cofactor (RicoF) or collagen binding $(\mathrm{CB})$ or ristocetin induced platelet aggregation (RIPA)]. This is counter-intuitive at first as the total vWF Antigen is often very high but it is caused by this imbalance between molar concentration and functional interaction between endothelia and platelets causing acquired von Willebrand's disease.

Mechanical assist devices will introduce turbulence as described above but also lead to further platelet consumption, platelet degranulation and may predispose to clot formation in areas of relative stasis. While more physiological, pulsatile flow introduces additional complexity and platelet trauma through compression of the blood by the roller pumps, the body's autoregulation and pressure sensing system has also been designed for pulsatile flow. Continuous flow has additional unintended consequences such as overstimulation of baroceptors and thus affecting microcirculation, increased risk of hemorrhagic stroke, increased matrix metalloproteinase expression, oxidative stress, and increased aortic stiffness (8). Hypoxic conditions are better tolerated when flow is pulsatile (11). Another pump related problem is heat generation. Heat, particularly when a clot affects the pump mechanism directly can be excessive and contribute both to haemolysis, thrombotic activation and consumption. Red cells break up may generate spurious thrombocytosis in automated cell counters. Changes in neutrophils can spuriously affect the automated differential white count causing an increase in measured eosinophils (12-16).

\section{The pump circuit surface treatment}

Circuit tubing is often coated with chemically active compounds or anticoagulants, also referred to as 'biomimetics', the most commonly used one remains heparin. There are a number of potential replacement coating materials in development that reduce contact factor activation such as the monoclonal antibody $3 \mathrm{~F} 732$ to factor XIIa or single-strand RNA molecules (aptamers) that interfere at different stages of contact/intrinsic cascade activation (11F7t 33 alone or in combination with R9d-14t, RNABA4, R4c-XII-1t, Kall1-T4 or FELIAP). Imitation of the endothelial release of nitrous oxide (NO) by utilising $\mathrm{N}$-diazeniumdiolates and potentially combining this with bonded direct thrombin inhibitors is another strategy. $\mathrm{NO}$ release is controlled by $\mathrm{pH}$ control locally within in the circuit and this does not cause systemic effects. This leads to conversion of endogenous S-nitrosothiols with the help of bonded copper ions into the circuit material. Another approach is to prevent interaction of the lining of the support device through passivation through phosphorylcholine, albumin and 2-methoxyethylacrylate (PMEA) (17,18). 
The utilisation of actual host endothelial cells or biocompatible endothelial cells could allow long term support in the future, but is not currently technically feasible to induce rapid coating of the central access cannulae or oxygenator tubing using the patient's stem cells (17-19).

\section{The anatomical site of the cannulisation and the circuit design}

Venous cannulation is associated with line infection and thromboembolism. Where in addition arteries are cannulated, injury to the thicker arterial wall can lead to embolization of plaque or calcium and artery dissection. In addition, the calibre of the catheter will cause a degree of obstruction in the artery distal to the insertion site, which can be associated with tissue ischaemia and compartment syndrome. Additional distal perfusion cannulae and sometimes surgical intervention (fasciotomy) can be required. Where the lung is bypassed rather than merely replaced in tandem to the patient's lung (e.g., in VA ECMO as opposed to VV ECMO), the relative risk of long-term mortality and morbidity is greatly increased. Even very small emboli from the outflow cannulae have the capacity to cause arterial embolisation. Other circuit designs such as the tandem heart similarly induce a new risk of paradoxical emboli by creating right left shunting as the inflow cannula is positioned by piercing the atrial septum.

While implantable MCAD are generally safer from a microbiological and thromboembolic perspective, the implantable devices have additional sources of infection such as the driveline which is tunnelled subcutaneously to the device to propel the internal mechanism while keeping the control element and battery outside the body.

For balloon pumps in particular improved balloon design and increasing miniaturisation have allowed insertion kits that do not require a sheath and so reducing the risk of dissection of the arterial wall.

\section{Duration}

In addition to the mechanical aspects of MCAD the intended duration of support is also important to understand the range of complications. At baseline MCAD are typically used in those with the most severe cardiac impairment associated with prolonged inotropic support, multiorgan failure and due to the emergency context usually also complex platelet-based coagulopathy. The implantable mechanical assist devices are the left ventricular assist device is intended for long-term use or even destination therapy. Line related bleeding complications are more likely to be encountered at the beginning of therapy whereas other complications such as GI bleeding due to AV malformations are long term complications caused by the continuous flow support.

\section{Bleeding}

Bleeding complications are common and the risk of bleeding varies greatly dependent on the type of circulatory support the patient is receiving and what other treatments the patient has received prior to MCAD support. Bleeding can broadly be classified into bleeding associated with cannulation or surgical implantation/intervention and spontaneous bleeding with no surgical cause. Where circulatory support was instituted as an emergency the history is often incomplete and particularly spontaneous bleeding and bleeding from the ear nose and throat region, upper gastrointestinal and urinary tract should prompt investigation for a congenital clotting factor deficiency with the help of a bleeding score and appropriate laboratory testing as well as considering acquired von Willebrand syndrome. A prolonged aPTT however may not only be caused by deficiency of the clotting factors VIII, IX and XI. Asymptomatic aPTT prolongation may also have been caused by other clotting factors that do not predispose to bleeding such as factor XII deficiency or isolated presence of Lupus anticoagulant antibodies.

Acquired von Willebrand syndrome due to the loss of high molecular weight vWF is believed to be one of the key mechanisms that is associated with excessive bleeding in patients with prolonged extracorporeal circuit therapy. vWF is cleaved by the action of the enzyme ADAMTS13. Von Willebrand multimers in health are protected from premature cleavage by ADAMTS13 through the adoption of a conformation that reduces the exposure of potential cleavage sites $(20,21)$.

As these assist devices consume particularly the high molecular weight vWF multimers gastrointestinal (GI) bleeding is common. Similar features are observed in subtypes of congenital vWD if there is absence of high molecular weight vWF (22). This in turn leads to a variable pattern of abnormal vWF function in vitro, e.g., predominantly affecting collagen binding.

There are only a few studies that have analysed the prevalence of reduced vWF factor level and activity. 
Generally a combination of vWF:Act $[($ RIcof $) /(\mathrm{CB})] /$ $\mathrm{vWF}$ :Antigen (vWF:Ag) of $<0.70$ is accepted as a diagnostic criterion. Where available Multimer analysis should be performed in addition to positively demonstrate the reduced concentration or absence of multimers. Prevalence varies according to the type of mechanical support with an unknown prevalence for the majority of devices. Small studies in ECMO have shown $85-100 \%$ prevalence.

The overall relevance of the in vitro phenomenon for clinical bleeding and other outcomes is unclear. Treatment checklists and algorithms based on extrapolation and expert opinion or retrospective single centre studies have been published. The main concern from a haematological perspective is that $\mathrm{vWF}: \mathrm{Ag}$ and $\mathrm{vWF}$ :Act are often $2-4 \times$ above the upper limit of normal and although the activity of the vWF lags behind it is still increased. Where Platelet aggregation is performed in addition to vWF:Act a suboptimal platelet aggregation maybe demonstrable (RIPA) in addition. However, these tests are time consuming and not always available when bleeding occurs.

In percutaneous and paracorporeal MCAD the most common bleeding complications affect the access lines or concomitantly inserted other percutaneous lines like chest drains (23).

In implantable intracorporeal MCAD the most common sites of bleeding are intracranial (1-9\%) and GI bleeding which can affect $5 \%$ to $40 \%$. AV malformations, angiodysplasia and ulcers due to concomitant antiplatelet therapy can predispose to bleeding. AV malformations are especially prevalent in patients with continuous flow pumps.

Nearly all patients with ventricular assist devices will develop abnormal vWF Act/Ag ratios, however In only 14$30 \%$ of patients VAD insertion is complicated by bleeding $(4,20)$. Geisen et al. found that $10 / 12$ of VAD recipients on day 1 showed acquired $\mathrm{vWD}$ and that all patients had acquired vWD by day 3 (24). The risk of bleeding is greatest in older patients, patients with cardiogenic shock, patients who are on anticoagulation or have liver dysfunction due to heart failure or kidney failure. The acquired vWD changes are reversible within $48 \mathrm{~h}$ when ventricular assist ceases.

In a small retrospective study of 73 patients undergoing surgery during VV ECMO, Holzer found only a difference in Fibrinogen between those patients receiving more than 2 units of blood vs. those who received fewer units of blood statistically significant (25).

The risk of bleeding in continuous flow VAD devices is reported to be ten times greater than in pulsatile devices with AV malformations being responsible in 20-60\% of cases of $\operatorname{VAD}(4,10,26)$, possibly caused by increased angiopoietin 2 levels (22). GI bleeding, however, is the most common VAD complication affecting 20-30\%, but is shared complication for all long-term continuous flow devices (27). Axial flow may cause a lesser degree of acquired vWD compared to the centrifugal design (28). In the HeartMate II device $(\mathrm{n}=145)$, Bunte et al. (29) found the peak incidence of bleeding to occur was within 2 weeks of implantation and then to be manifest as thoracic, mediastinal or GI bleeding. The reintroduction of pulsatility in the HeartMate $\mathrm{III}^{\oplus}$ design has not significantly reduced the frequency of GI bleeding despite reduced consumption of high molecular weight $\mathrm{vWF}(8,30)$.

Treatment is of MCAD associated acquired vWD deficiency is controversial.

Kalbhenn et al. have published a single centre experience-based transfusion algorithm with the thresholds for factor replacement on ECMO. In this paper they advocate aggressive correction of $\mathrm{vWF}: \mathrm{Ag} / \mathrm{vWF}$ :Act based on activity, factor VIII and factor XIII as well as fibrinogen of $1.5 \mathrm{~g} / \mathrm{dL}$ (31).

The treatment with vWF concentrate is often not successful due to mechanical consumption of the replacement product. vWF concentrate will increase the vWF:Ag more than the activity and increase the patient's factor VIII level so increasing the risk of thrombosis. Notably GI bleeding is also a $7 \times$ risk factor for stroke, likely through interruption of anticoagulation rebleeding rates after restarting anticoagulation are high (4).

The neoangiogenesis is a novel target for bleeding prevention with the use of ACE inhibitors or angiotensin receptor blockers in the first $30 \mathrm{~d}$ after implantation. Another target is the administration of omega-3-fatty acids which have been shown to reduce the risk of bleeding at a dose of several $g$ daily in a retrospective study. Octreotide and thalidomide have also been tried off label with some success in small retrospective case series of LVAD bleeding. In vWD in the absence of MCAD in addition there are reports of successful use of Danazol for GI bleeding (21). There is a small cohort where Doxycylin has been used reduce ADAMTS13 activity and improve the vWF halflife $(27,32)$ but it is yet to be tested in patients with LVAD placement.

\section{Platelet phenotype}

The exposure of the blood to the circuit material leads 
to the deposition of platelets on the circuit surface. This process begins within minutes of commencing mechanical support and is associated with clotting factor and contact factor activation and depletion. The number of platelets that take part in coating the surface increases with increasing shear force (4). In addition to the tubing system another area that attracts major platelet deposition is the pump itself. Where clots form in this area they typically contain fibrin cores with vWF deposited in the outer layers in association with platelets $(20,33,34)$.

Platelet aggregometry shows a global reduction compared to pre ECMO (34). Platelets during ECMO therapy typically decrease in numbers and show altered expression of adhesion molecules such as GP VI, GPI $\alpha$, CD62, CD63 and $\beta$-thromboglobulin. Transient changes in platelet factor 4 which recover over the following 7 days have also been described $(4,34)$.

In ventricular assist devices pulsatile devices seem to show more platelet activation than continuous flow devices, whereas the continuous flow devices show evidence of persistent endothelial activation.

Intracorporeal pulsatile devices show activation of CD63, p-selectin and thrombospondin highest after implantation but increased still at 3 weeks, whereas paracoporeal pulsatile VAD patients were found to have abnormal p-selectin expression but not CD63 or thrombospondin (35). In the MicroMed $^{\circledR}$ (Micromed Technology Inc., Houston, Texas, USA) continuous flow device p-selectin, CD63 and CD31 was initially elevated but returned to normal after 4 weeks, the Jarvik $2000^{\circledR}$ (Jarvik Heart Inc, New York, NY, USA) and HeartMate $\mathrm{II}^{\circledast}$ did not show activation of these markers (35). These markers have not been linked to clinical symptoms of thrombosis or bleeding yet (35).

Reactive oxygen species mitochondrial membrane potential and markers of apoptosis were found increased in VAD (Jarvik2000 ${ }^{\circledR}, \mathrm{HVAD}^{\circledR}$ and HeartMate $\mathrm{II}^{\circledR}$ ) patients and in a small group of HeartMate $\mathrm{II}^{\circledR}$ patients to be linked to bleeding complications (35).

It is likely also the consequence of a negative selection mechanism where the most reactive platelets adhere to catheters and pump surfaces and only less reactive platelets remain in circulation which in turn leads to a mixed platelet secretion defect affecting alpha and delta granules (36).

Shear stress also induces shedding of GP Ib which again if observed in the first 2 weeks may correlate to clinical bleeding phenotype but other receptors such as GP VI and GP IIb/IIIa also show shear force related shedding (35). Genetic polymorphisms such as of GpIIb/IIIa may also have an influence on complications (35). Increases in interactions with monocytes may also be important through formation of monocyte platelet complexes with increased expression of CD14 (35). In a small cohort of 15 patients with ventricular assist device support these patients had an increased frequency of embolic complications (35). Patients in the early phase post LVAD insertion were found to have increased platelet microparticles with increased levels of IL4, IL6, I-CAM-1, E-selectin and VCAM-1. These elevations were persistent but less pronounced at 6 months (35).

\section{Anticoagulation}

The initial insertion of MCAD devices follows protocols with significant variation between institutions $(7,37)$. Most centres agree that haemostasis at the time of implantation should be normalised temporarily (21). Following insertion all of these devices are initially managed with a combination of unfractionated heparin (UFH) around the time of implantation with the majority of them having manufacturer's recommendations to continue with heparinisation while they are in situ.

The 2013 International Society for Heart and Lung Transplantation (ISHLT) guidelines (38) recommend commencement of UFH or alternative anticoagulant within $2 \mathrm{~d}$ of implantation of ventricular assist devices. Those devices that can remain for more than just a few days typically transfer to oral anticoagulation with warfarin with an INR of 2.0 (HeartMate II) or 2.5-3.0 (HVAD). VAD insertion however has a high rate of reoperation for bleeding which can be as high as $30 \%$ which is why there are ongoing efforts to make the initial anticoagulation less aggressive.

The newer HeartMate III VAD was found to have a significantly lower rate of pump thrombosis compared to the HeartMate II device at 6 months in the Momentum 3 RCT trial (0.7 vs. $7.7 \%, \mathrm{P}=0.002)$ with comparable rates of bleeding or stroke (27).

Centres who have initiated warfarin loading without UFH but with aspirin and warfarin alone after device implantation of the HeartMate $\mathrm{II}^{\circledR}$ device have not seen a noticeable increase in thrombotic evens in the short term (27).

Conversely however the reduced intensity of bridging anticoagulation following the initial insertion period due to increased frequency of GI bleeding with the centrifugal flow devices has seen an increase of pump thrombosis at 3 months for the HeartMate II ${ }^{\circledR}$ VAD (39). (incidence up 
from levels of $2.2 \%$ (in 2011) to levels as high as $8.4 \%$ (in 2013) (27).

The prevention of pump thrombosis relies on mechanical and non-mechanical factors and only those HeartMate $\mathrm{II}^{\circledR}$ patients that had pump speeds $>9,000 / \mathrm{min}$, had consistent bridging, followed all surgical recommendations had $1.9 \%$ vs. $8.9 \%$ incidence of pump thrombosis in the PREVENT trial, which is referred to as the three $\mathrm{P}$ factors (patients, pumps and pills) $(2,40)$.

The use of concomitant antiplatelet therapy varies. Heartmate I, HVAD and HeartMate II devices in addition are treated with aspirin by some centres $(7,37)$. Some of the older designs in addition recommend dipyridamole dual antiplatelet therapy and some only during the first 4 weeks.

Novel approaches are to measure the degree of platelet blockade in vitro (e.g., Platelet mapping ${ }^{\circledR}$ ) warfarin alone and reduced intensity warfarin with an INR of 1.6-2.0 $(27,41,42)$.

In LVAD thrombin generation between warfarin treated patients with Aspirin or without Aspirin showed identical ETP but marked differences in peak and lag time (43).

Direct oral anticoagulants (DOACs) have not been shown to equivalent to warfarin in VAD patients. In one study dabigatran had comparable efficacy to warfarin and less major bleeding, another trial switching from warfarin to dabigatran after 4 weeks had to be discontinued prematurely due excess thromboembolic events (dabigatran $110 \mathrm{mg} \mathrm{BD}$ ) $(4,27)$.

Bridging is used frequently for subtherapeutic INRs but the threshold is not universal and some centres do not have an agreed lower limit below which LMWH would be given and practice varies depending on device and patient's history (37).

If MCAD associated venous thrombosis occurs, consistent uninterrupted anticoagulation may be sufficient to reduce the clot burden but in some case repositioning of the line that is associated with the clot may be required.

\section{Venous thromboembolism and arterial embolic disease}

Stroke occurs in VAD with an annualised risk of 7-10\% (4). Strokes are complicated by a $12 \%$ fatality rate. Stroke risk reduction is achieved by heparinisation, warfarin therapy and possible additional anti-platelet therapy with aspirin and clopidogrel/dipyridamole but practice varies between centres and devices.

Large strokes may necessitate interruption of anticoagulation therapy for a variable duration of time if the clinicians are concerned about the risk of haemorrhagic transformation of the stroke.

Antithrombin (AT3) deficiency is highly prevalent around the time of MCAD insertion due sepsis, consumption, critical illness or hepatic impairment (41). In a meta-analysis of 20 AT3 replacement trials in patients on ICU it was shown to be associated with a $1.5 \times$ increased risk of bleeding but with no effect on mortality. There are a number of lower quality case series that report no increase in bleeding. It is important therefore to conduct further trials about the relative contribution of antithrombin replacement for VTE prophylaxis (2).

Pump thrombosis can affect the inflow cannula, pump and outflow cannula. Pump thrombosis should be suspected if two of the following criteria coexist: (I) abnormal pump parameters (pump power elevation); (II) raised LDH or plasma-free haemoglobin; (III) new heart failure symptoms unexplained by structural abnormality. Pump thrombosis has a high mortality with a survival of $65 \%$ after a first pump replacement and only $50 \%$ after a second.

The treatment of pump thrombosis can be medical or surgical dependent on location of the clot, overall prognosis for the patient and institutional experience. Medical therapy typically consists of a combination of UFH, thrombolysis and possibly GP IIb/IIIa blockers. Where case series have been reported the success rates are low-between $25-50 \%$ resolution of clot and a mortality of up to $17-52 \%$. Bleeding complications are observed in up to $65 \%$ of patients with increasing risk if thrombolysis is employed. Pump thrombosis especially if it affects the pumping mechanism itself is associated with haemolysis $(4,44,45)$. A weak point of pump design are the rotor bearings which can be liable to heat generation if based on hydrodynamic or mechanical principles. Magnetically levitated impellers however may increase the dwell time of the blood in areas although the friction is much reduced. Pump thrombosis can also be secondary to aspiration of pannus material and cause embolic complications (40).

The incidence of HIT is low and for ECMO is cited at $0.36 \%$. Not all studies have diagnosed HIT with functional assays leading to variation in the reports on incidence and prognosis. Where HIT is diagnosed care must be taken to avoid further heparin exposure and the patient should be switched to non-heparin coated circuit and pump material (46).

Nafamostat mesylate (NM) (47) has been used in small case series in paediatric and adult patient for anticoagulation during ECMO support with conflicting results. It is a proteinase inhibitor with off target effects on renal 
Table 1 Comparison of the clinical and laboratory features of immune haemolysis (auto-immune and allo-immune) and mechanical haemolysis

\begin{tabular}{llll}
\hline Haemolysis & Auto-immune haemolytic anaemia & Allo-immune haemolytic anaemia & Non-immune \\
\hline Blood film & Spherocytes or auto-agglutination & Spherocytes or auto-agglutination & Red cell fragmentation \\
LDH & Raised +++ & Raised + & Raised +++ \\
Haptoglobin & Absent, if detectable likely & Absent, if detectable likely extra-vascular & Absent \\
& extra-vascular haemolysis & haemolysis & Indirect bilirubin $++/$ \\
Liverfunction tests & Indirect bilirubin $++/$ & Indirect bilirubin $++/$ & Direct bilirubin + \\
& Direct bilirubin + & Direct bilirubin + & Detectable \\
Free haemoglobin & Detectable & Only in very brisk haemolysis & Detectable \\
Urine haemosiderin & Detectable & Detectable & Not causally related to transfusion \\
& Not causally related to transfusion & History of transfusion in last $6 / 52$ & Often concurs however \\
\hline
\end{tabular}

potassium excretion. Due to the non-cross reactivity with heparin this could be a promising anticoagulant in heparin intolerant patients.

\section{Haemolysis}

Haemolysis may be broadly categorised in immune and non-immune haemolysis. Immune haemolysis typically is caused by allo-antibodies in response to previous transfusion (Table 1).

The blood film may allow initial stratification. Presence of spherocytes or autoagglutination may indicate immune haemolysis whereas the presence of fragments may indicate mechanical haemolysis e.g., due to excessive flow velocities or pump thrombosis. $\mathrm{LDH}$ and haptoglobin allow an assessment of the briskness of haemolysis, similarly increased total and indirect bilirubin can indicate haemolysis. The ratio between direct and indirect bilirubin may further help to identify haemolysis as a cause but its interpretation is often limited by coexistent hepatic impairment. Haptoglobin is useful to try to distinguish between intra and extra-vascular haemolysis and would be expected to be absent in the presence of significant MCAD related intravascular haemolysis as well if there is brisk autoimmune haemolysis. The LDH further helps to assess the degree of intravascular haemolysis. The LDH however is not specific for haemolysis however is advocated by some to be a very reliable measure and changes over time can indicate pump thrombosis. In Immune haemolysis while oxygen delivery becomes less efficient with anaemia, no effect on the pump load would be expected. Red cells too are subjected to extreme shear forces and focal points of increased temperature. The shear force exerted on passing blood increases with reducing calibre which is why haemolysis is associated with small leaks and thrombosis.

An increase in device power which is based on the Watt's law relationship of power $=$ voltage (pressure gradient) $\times$ current (flow) means that a clot in the pump mechanism will lead to a marked increase in power load to maintain device flow and thus causing haemolysis through the change in pressure and velocity across a reduced cross section (7). The impella device is associated with haemolysis in $60 \%$ of patients who requires support for $>24 \mathrm{~h}$ (4). The speed of the centrifugal pump is markedly greater which combined with a much narrower calibre. Haemolysis is quantified with a combination of free haemoglobin, LDH and Haptoglobin. Confounding can occur due to rhabdomyolysis or hyperbilirubinemia (4). Haemosiderin in urine maybe a useful adjunctive test to confirm intravascular haemolysis.

Management will depend on the cause. Centrifugal, axial and pulsatile devices show different susceptibilities to pathophysiological changes causing changes in head pressure. Axial flow devices such as the HeartMate II show an increase in negative pressure is there are changes in the pressure gradient between the ventricle and the aortic arch that centrifugal devices are insensitive to. The suction can aspirate part of the ventricle wall and the reduction in inflow cannula diameter can be associated with haemolysis (7). Mechanical haemolysis should prompt investigation of the cause of haemolysis and an attempt at correction. Where the cause is not evident changes in flow or cannula position 
should be considered. Where this is not an option careful monitoring should be instituted and it is important to note that chronic haemolysis can predispose to VTE and pulmonary hypertension.

Allo-immune haemolysis is usually self-limiting and requires very minimal intervention. Careful antibody identification and sparing transfusion should be instituted. Only about $4 \%$ of patients who receive transfusions develop antibodies. In the acute phase of a delayed haemolytic transfusion reaction there may not be free detectable alloantibody until all allogeneic red cells have been destroyed.

Autoimmune haemolysis may require aggressive intervention with a prolonged course of steroids but would be exceedingly unlikely in a patient receiving MCAD. A confounder is the fact that approximately $10 \%$ of patients have a positive direct antiglobulin (Coombs') without being clinically affected by haemolysis, emphasizing that the intensity of the patient's haemolysis cannot be derived from laboratory parameters alone.

\section{Anaemia, causes of, transfusion alternatives $(48,49)$}

Oxygen delivery is a function of haemoglobin, cardiac output, haemoglobin oxygen saturation and partial pressure of $\mathrm{O}_{2}$ in the plasma. Delivery of oxygen is approximately $2-5 \times$ of oxygen consumption in homeostasis. Oxygen consumption can therefore be maintained until oxygen delivery falls to $2 \times$ or less of oxygen consumption (11). The oxygen content of a volume of blood is dependent on $\mathrm{pO}_{2}$ and haemoglobin content with the saturation levels that are achieved directly proportionate to the concentration of haemoglobin. Reduced oxygen delivery per $\mathrm{ml}$ of blood can be compensated for by increasing cardiac output but ultimately anaerobic metabolism results.

Transfusion in cardiac ICU should ideally be driven by oxygen delivery and cardiac oxygen demand rather than a set $\mathrm{Hb}$ level.

Every additional gram of haemoglobin increases the delivery of oxygen however at the cost of increasing viscosity and heart strain. Conversely the ICU patient is usually at rest meaning that the oxygen extraction from the blood will be reduced and a gain in $\mathrm{Hb}$ concentration will not linearly increase the amount oxygen extracted but may allow to tolerate a reduction in cardiac output more easily. The above is borne out in the few studies that are available regarding restrictive and liberal transfusion strategy.

In multicentre observational study (50), patients who received transfusions had excess mortality overall and in ICU. ICU stay also was significantly longer in the transfused group (7.2 vs. $2.6 \mathrm{~d}$ ) This remained so even after sequential organ failure assessment (SOFA) score matching and at all admission haemoglobin levels. Transfusion decisions were left to individual clinicians in 146 ICUs who did not apply a unified threshold.

In a small $(\mathrm{n}=167)$ single centre observational cohort study with $54 \%$ cardiac patients (51) treated with a transfusion threshold of $70 \mathrm{~g} / \mathrm{L}$ there was excess mortality, worse SOFA score on day 28, longer ICU and hospital stay in the transfused group which reached statistical significance and transfusion was a significant independent risk factor in multivariate analysis for mortality.

In a systematic review in critically ill patient Marik et al. (52) included 272,596 patients in 45 studies and found that the risk outweighed the benefits of red cell transfusion in 42 of 45 , was neutral in 2 and was of patient benefit in one in the subgroup of the elderly and acute myocardial infarction, there with a haematocrit threshold of $30 \%$ (which would convert to approximately $\mathrm{Hb}<100 \mathrm{~g} / \mathrm{L}$ ).

The original randomised controlled transfusion requirements in critical care (TRICC) trial that led to advocating a restrictive over a liberal transfusion strategy was conducted in a general ICU and included only $20 \%$ cardiac patients. The two transfusion thresholds trialled against one another were 70 and $100 \mathrm{~g} / \mathrm{L}$ (49). There was a trend to lower 30 day mortality and ICU mortality in the overall cohort. 30-day mortality became significant only in the under 55 years old and those with an APACHEII score of $\leq 20$. The restrictively transfused cardiac patient did not show the same trend in mortality difference as the other cohorts. When the same strategy was employed in septic ICU patients (TRISS) trial no significant difference in $90 \mathrm{~d}$ mortality or organ support was identified between a transfusion threshold of $90 \mathrm{~g} / \mathrm{L}$ and $70 \mathrm{~g} / \mathrm{L}$. Cardiac patients made up $14 \%$ of TRISS patients.

Carson et al. (53) conducted a meta-analysis of 12,587 patients in 31 trials comparing the transfusion triggers of 70-90 g/L against $90-100 \mathrm{~g} / \mathrm{L}$ and found no effect on the 30 day mortality and other assessed outcomes (cardiac events, stroke, thromboembolism or infection) although the risk of transfusion was significantly reduced in patients treated with a restrictive strategy.

Holst et al. (54) included 9,813 patients in 31 trials with no effect on mortality, myocardial infarction, cardiac events or overall morbidity. Transfusion triggers again varied between $70-97 \mathrm{~g} / \mathrm{L}$ in Holst's restrictive group.

Docherty et al.'s meta-analysis (55) of 3,033 cardiac 


\section{Page 10 of 13}

patients not undergoing surgery in 11 trials showed no clear detriment with a restrictive strategy on 30-day mortality but an increased risk of acute coronary events (RR 1.78, 95\% CI: 1.18-2.7, $\mathrm{P}=0.01$ ) . Docherty's study was not limited to ICU patients.

In an observational study of 1711 ICU patients, the risk of infection was $15.38 \%$ vs. $2.93 \%$ in the transfused groups $v s$. untransfused. Each unit of blood received increase the risk of nosocomial infection by factor 1.5 with a mean number of units received in the transfused group of 4 (49).

\section{Transfusion alternatives}

Despite widespread presence of iron restriction in up to $35 \%$ ICU patients iron infusions were not shown to reduce transfusion dependence, length of stay, mortality or even $\mathrm{Hb}$ at 10 days or the end of follow-up in a meta-analysis of 665 patients in RCTs $(49,56)$.

\section{Erythropoietin}

When measured EPO levels were found to be relatively decreased compared to other patients with anaemia in ICU patients and although a modest reduction in transfusion requirement can be demonstrated when iron and erythropoietin are combine, there was no benefit in mortality or length of stay which is why the 2012 British Commission for Standards in Haematology guidelines advised against the use of EPO in ICU patients and advised the adoption of a transfusion threshold of $70 \mathrm{~g} / \mathrm{L}$ instead (57). Adatya et al. has demonstrated in a meta-analysis of 944,856 patients in 48 studies a significant increased risk of thromboembolic events in EPO treated critically ill patients. EPO use has also been found to be associated with a $2.4 \times$ increased risk of LVAD thrombosis (42).

\section{Conclusions}

There are a large number of different cardiac assist devices in clinical use. They share a number of complications and despite great advances in design and experience still are often complicated by GI bleeding and pump thrombosis or systemic embolization. New biomaterials may allow safe reduction in anticoagulation and a better understanding of how to prevent the formation of $\mathrm{AV}$ malformation may help in the future make devices safer.

\section{Acknowledgments}

Funding: None.

\section{Besser. Bleeding, haemolysis and coagulation in MCAD patients}

\section{Footnote}

Provenance and Peer Review: This article was commissioned by the Guest Editor (Kamen Valchanov) for the series "Perioperative Management of Patients with undergoing Mechanical Circulatory Support" published in Annals of Translational Medicine. The article was sent for external peer review organized by the Guest Editor and the editorial office.

Conflicts of Interest: The author has completed the ICMJE uniform disclosure form (available at http://dx.doi. org/10.21037/atm-20-405). The series "Perioperative Management of Patients with undergoing Mechanical Circulatory Support" was commissioned by the editorial office without any funding or sponsorship. MWB reports personal fees from Novartis, personal fees from STAGO, personal fees from Cosmopharma, personal fees from Werfen, during the conduct of the study. The author has no other conflicts of interest to declare.

Ethical Statement: The author is accountable for all aspects of the work in ensuring that questions related to the accuracy or integrity of any part of the work are appropriately investigated and resolved.

Open Access Statement: This is an Open Access article distributed in accordance with the Creative Commons Attribution-NonCommercial-NoDerivs 4.0 International License (CC BY-NC-ND 4.0), which permits the noncommercial replication and distribution of the article with the strict proviso that no changes or edits are made and the original work is properly cited (including links to both the formal publication through the relevant DOI and the license). See: https://creativecommons.org/licenses/by-nc-nd/4.0/.

\section{References}

1. Kirklin JK, Naftel DC, Pagani FD, et al. Seventh INTERMACS annual report: 15,000 patients and counting. J Heart Lung Transplant 2015;34:1495-504.

2. Raffini L. Anticoagulation with VADs and ECMO: Walking the tightrope. Hematology Am Soc Hematol Educ Program 2017;2017:674-80.

3. NHSBT. Heart Transplant Waiting List Grows. 2019. Available online: https://www.organdonation.nhs.uk/ get-involved/news/heart-transplant-waiting-list-grows/. Accessed 29/12/2019. 
4. Hilal T, Mudd J, DeLoughery TG. Hemostatic complications associated with ventricular assist devices. Res Pract Thromb Haemost 2019;3:589-98.

5. Bortot M, Ashworth K, Sharifi A, et al. Turbulent Flow Promotes Cleavage of VWF (von Willebrand Factor) by ADAMTS13 (A Disintegrin and Metalloproteinase With a Thrombospondin Type-1 Motif, Member 13). Arterioscler Thromb Vasc Biol 2019;39:1831-42.

6. Sen A, Larson JS, Kashani KB, et al. Mechanical circulatory assist devices: a primer for critical care and emergency physicians. Crit Care 2016;20:153.

7. Blum FE, Weiss GM, Cleveland JC Jr, et al. Postoperative Management for Patients With Durable Mechanical Circulatory Support Devices. Semin Cardiothorac Vasc Anesth 2015;19:318-30.

8. Castagna F, Stohr EJ, Pinsino A, et al. The Unique Blood Pressures and Pulsatility of LVAD Patients: Current Challenges and Future Opportunities. Curr Hypertens Rep 2017;19:85.

9. Engelke J, Karmonik C, Rengier F, et al. Competing Flow Between Partial Circulatory Support and Native Cardiac Output: A Clinical Computational Fluid Dynamics Study. ASAIO J 2018;64:636-42.

10. Wever-Pinzon O, Selzman CH, Drakos SG, et al. Pulsatility and the risk of nonsurgical bleeding in patients supported with the continuous-flow left ventricular assist device HeartMate II. Circ Heart Fail 2013;6:517-26.

11. Annich G. editor. ECMO: extracorporeal cardiopulmonary support in critical care, 4th ed. Extracorporeal Life Support Organization, 2012.

12. Nagelkirk PR, Hogan KB, Hoare JM. Ambient temperature affects thrombotic potential at rest and following exercise. Thromb Res 2012;130:248-52.

13. Bennett V. The spectrin-actin junction of erythrocyte membrane skeletons. Biochim Biophys Acta 1989;988:107-21.

14. Gershfeld NL, Murayama M. Thermal Instability of Red Blood Cell Membrane Bilayers: Temperature Dependence of Hemolysis. J Membr Biol 1988;101:67-72.

15. Choi JW, Pai SH. Changes in Hematologic Parameters Induced by Thermal Treatment of Human Blood. Ann Clin Lab Sci, 2002;32:393-8.

16. Yost G, Joseph CR, Royston T, et al. Heat Generation in Axial and Centrifugal Flow Left Ventricular Assist Devices. ASAIO J 2016;62:684-9.

17. Eytan D, Bitterman Y, Annich GM. VV extracorporeal life support for the Third Millennium: will we need anticoagulation? J Thorac Dis 2018;10:S698-S706.
18. Chabata CV, Frederiksen JW, Sullenger BA, et al. Emerging applications of aptamers for anticoagulation and hemostasis. Curr Opin Hematol 2018;25:382-8.

19. Sotiri I, Robichaud M, Lee D, et al. BloodSurf 2017: News from the blood-biomaterial frontier. Acta Biomater 2019;87:55-60.

20. Malfertheiner MV, Pimenta LP, Bahr VV, et al. Acquired von Willebrand syndrome in respiratory extracorporeal life support: a systematic review of the literature. Crit Care Resusc 2017;19:45-52.

21. Leebeck FWG, Muslem R. Bleeding in critical care associated with left ventricular assist devices: pathophysiology, symptoms, and management. Hematology Am Soc Hematol Educ Program 2019;2019:88-96.

22. Leebeek FWG, Atiq F. How I manage severe von Willebrand disease. Br J Haematol 2019;187:418-30.

23. Lotz C, Streiber N, Roewer N, et al. Therapeutic Interventions and Risk Factors of Bleeding During Extracorporeal Membrane Oxygenation. ASAIO J 2017;63:624-30.

24. Geisen U, Heilmann C, Beyersdorf F, et al. Non-surgical bleeding in patients with ventricular assist devices could be explained by acquired von Willebrand disease. Eur J Cardiothorac Surg 2008;33:679-84.

25. Holzer F, Gruber M, Philipp A, et al. Predictos of bleeding in ECMO patients undergoing surgery. Minerva Anestesiol 2020;86:47-55.

26. Crow S, John R, Boyle A, et al. Gastrointestinal bleeding rates in recipients of nonpulsatile and pulsatile left ventricular assist devices. J Thorac Cardiovasc Surg 2009;137:208-15.

27. Bader F, Mallah S, Atallah B. Choosing the best antithrombotic regimen in patients with ventricular assist devices. Curr Opin Cardiol 2020;35:162-9.

28. Lalonde SD, Alba AC, Rigobon A, et al. Clinical differences between continuous flow ventricular assist devices: a comparison between HeartMate II and HeartWare HVAD. J Card Surg 2013;28:604-10.

29. Bunte MC, Blackstone EH, Thuita L, et al. Major bleeding during HeartMate II support. J Am Coll Cardiol 2013;62:2188-96.

30. Bansal A, Uriel N, Colombo PC, et al. Effects of a fully magnetically levitated centrifugal-flow or axial-flow left ventricular assist device on von Willebrand factor: A prospective multicenter clinical trial. J Heart Lung Transplant 2019;38:806-16.

31. Kalbhenn J, Wittau N, Schmutz A, et al. Identification 
of acquired coagulation disorders and effects of targetcontrolled coagulation factor substitution on the incidence and severity of spontaneous intracranial bleeding during veno-venous ECMO therapy. Perfusion 2015;30:675-82.

32. Bartoli CR, Kang J, Restle DJ, et al. Inhibition of ADAMTS-13 by Doxycycline Reduces von Willebrand Factor Degradation During Supraphysiological Shear Stress: Therapeutic Implications for Left Ventricular Assist Device-Associated Bleeding. JACC Heart Fail 2015;3:860-9.

33. Yaw HP, Van Den Helm S, MacLaren G, et al. Platelet Phenotype and Function in the Setting of Pediatric Extracorporeal Membrane Oxygenation (ECMO): A Systematic Review. Front Cardiovasc Med 2019;6:137.

34. Balle CM, Jeppesen AN, Christensen S, et al. Platelet Function During Extracorporeal Membrane Oxygenation in Adult Patients: A Systematic Review. Front Cardiovasc Med 2018;5:157.

35. Koliopoulou A, McKellar SH, Rondina M, et al. Bleeding and thrombosis in chronic ventricular assist device therapy: focus on platelets. Curr Opin Cardiol 2016;31:299-307.

36. Geisen U, Brehm K, Trummer G, et al. Platelet Secretion Defects and Acquired von Willebrand Syndrome in Patients With Ventricular Assist Devices. J Am Heart Assoc 2018. doi: 10.1161/JAHA.117.006519.

37. Jennings DL, Horn ET, Lyster H, et al. Assessing Anticoagulation Practice Patterns in Patients on Durable Mechanical Circulatory Support Devices: An International Survey. ASAIO J 2016;62:28-32.

38. Feldman D, Pamboukian SV, Teuteberg JJ, et al. The 2013 International Society for Heart and Lung Transplantation Guidelines for mechanical circulatory support: executive summary. J Heart Lung Transplant 2013;32:157-87.

39. Kirklin JK, Naftel DC, Kormos RL, et al. Interagency Registry for Mechanically Assisted Circulatory Support (INTERMACS) analysis of pump thrombosis in the HeartMate II left ventricular assist device. J Heart Lung Transplant 2014;33:12-22.

40. Massicotte P, Snyder T, Stulak J, et al. Ventricular assist device thrombosis: Mind your P's \&Q's-Pumps, patients, and pills. J Thorac Cardiovasc Surg 2017;153:1503-6.

41. Esper SA, Welsby IJ, Subramaniam K, et al. Adult extracorporeal membrane oxygenation: an international survey of transfusion and anticoagulation techniques. Vox Sang 2017;112:443-52.

42. Adatya S, Bennett MK. Anticoagulation management in mechanical circulatory support. J Thorac Dis 2015;7:2129-38.
43. Consolo F, Pozzi L, Pieri M, et al. Influence of Different Antithrombotic Regimens on Platelet-Mediated Thrombin Generation in Patients with Left Ventricular Assist Devices. ASAIO J 2020;66:415-22.

44. Matthews JC, Pagani FD, Haft JW, et al. Model for endstage liver disease score predicts left ventricular assist device operative transfusion requirements, morbidity, and mortality. Circulation 2010;121:214-20.

45. Pagano D, Milojevic M, Meesters MI, et al. 2017 EACTS/EACTA Guidelines on patient blood management for adult cardiac surgery. Eur J Cardiothorac Surg 2018;53:79-111.

46. Pollak U. Heparin-induced thrombocytopenia complicating extracorporeal membrane oxygenation support: Review of the literature and alternative anticoagulants. J Thromb Haemost 2019;17:1608-22.

47. Lim JY, Kim JB, Choo SJ, et al. Anticoagulation During Extracorporeal Membrane Oxygenation; Nafamostat Mesilate Versus Heparin. Ann Thorac Surg 2016;102:534-9.

48. Kim HS, Park S. Blood Transfusion Strategies in Patients Undergoing Extracorporeal Membrane Oxygenation. Korean J Crit Care Med 2017;32:22-8.

49. Jandu AS, Vidgeon S, Ahmed N. Anaemia and transfusion triggers in critically ill patients - What we have learnt thus far. J Intensive Care Soc 2019;20:284-9.

50. Vincent JL, Baron JF, Reinhart K, et al. Anemia and blood transfusion in critically ill patients. JAMA 2002;288:1499-507.

51. Silva Junior JM, Rezende E, Amendola CP, et al. Red blood cell transfusions worsen the outcomes even in critically ill patients undergoing a restrictive transfusion strategy. Sao Paulo Med J 2012;130:77-83.

52. Marik PE, Corwin HL. Efficacy of red blood cell transfusion in the critically ill: a systematic review of the literature. Crit Care Med 2008;36:2667-74.

53. Carson JL, Stanworth SJ, Roubinian N, et al. Transfusion thresholds and other strategies for guiding allogeneic red blood cell transfusion. Cochrane Database Syst Rev 2016;10:CD002042.

54. Holst LB, Petersen MW, Haase N, et al. Restrictive versus liberal transfusion strategy for red blood cell transfusion: systematic review of randomised trials with meta-analysis and trial sequential analysis. BMJ 2015;350:h1354.

55. Docherty AB, Walsh TS. Anemia and blood transfusion in the critically ill patient with cardiovascular disease. Crit Care 2017;21:61.

56. Shah A, Roy NB, McKechnie S, et al. Iron 
supplementation to treat anaemia in adult critical care patients: a systematic review and meta-analysis. Crit Care 2016;20:306.

Cite this article as: Besser MW. Post-operative of bleeding, haemolysis and coagulation in mechanical circulatory support patients. Ann Transl Med 2020;8(13):832. doi: 10.21037/atm-20405
57. Retter A, Wyncoll D, Pearse R, et al. Guidelines on the management of anaemia and red cell transfusion in adult critically ill patients. Br J Haematol 2013;160:445-64. 